\title{
Development of FEM - Programs for Modeling the Processes of Synthesis by Powder Metallurgy
}

\author{
Igor N. Sachkov ${ }^{1, \text { a) }}$, Viktoria F. Turygina, ${ }^{1, b)}$, Vitaly Ford ${ }^{2, c)}$, Anatoly P. \\ Shamanov ${ }^{1)}$, Oleg E. Khorev ${ }^{1)}$ \\ ${ }^{1}$ Ural Federal University, Mira Street, 19, Yekaterinburg, Russia, 620002 \\ ${ }^{2}$ Arcadia University, 450 S. Easton Road, Glenside, PA 19038, USA \\ a)insatch@e1.ru \\ b)Corresponding author: v.f.volodina@urfu.ru \\ c)fordv@arcadia.edu
}

\begin{abstract}
The finite element method (FEM) analyzes the features of the spatial distribution of Joule heat in two-phase matrix systems with round inclusions. It was found that in the processes of sintering peaks of heat generation power are formed at certain points of the medium. It is proposed to use the observed phenomenon for the formation of anisotropic and gradient materials.
\end{abstract}

Keywords: finite element method, powder metallurgy, Joule heat, material anisotropy, gradient materials

\section{INTRODUCTION}

One of the methods of powder metallurgy is the technology of synthesis through heating by flowing electric current [1]. Earlier, the heterogeneities of heat release in resistive materials were analyzed using analytical methods [2]. It was shown that if the metal contains topological defects of the structure, impurities, and other macroscopic inhomogeneity, then they become centers of intense release of Joule heat. The resulting "thermal structure" can lead, in particular, to thermal stresses that destroy the material. In the cited work, a one-dimensional, spherically symmetric process was studied. It can be expected that in the processing of sintering powder materials by flowing electric current, the discussed effects of the occurrence of thermal structure are also realized. In [3], the topology and spectra of released Joule power in two-phase resistive materials were studied using the finite element method.

This paper considers the possibility of using the finite element method (FEM) to create new technologies for the synthesis of gradient materials using powder anisotropy metallurgy.

\section{CALCULATION METHOD}

Consider the process of synthesizing a two-phase material representing a matrix containing inclusions of a round shape. We take into account that the conductance of the phases differ significantly in their specific electrical resistance. The real analogue of the objects under discussion can be copper-tungsten contact materials. Figure 1 shows the idealized structure of the material under discussion. We confine ourselves to a two-dimensional stationary picture of the process. We will assume the presence of flat symmetry, that is, we assume that the round inclusions in cross section have a generally cylindrical shape.

Consider the thermal effect of electric current, the direction of which is shown in Fig. 1 arrow. To this end, it is advisable to apply in this work an approach developed in [3-5], based on the use of the finite element method.

Let us select in the system under consideration an elementary square cell, whose boundaries are formed by the adiabatic and isopotential planes of the regular system under consideration [3-5]. The cell construction diagram is shown in Fig.2. We use the dimensionless variables $\mathrm{x}$ and $\mathrm{y}$. 


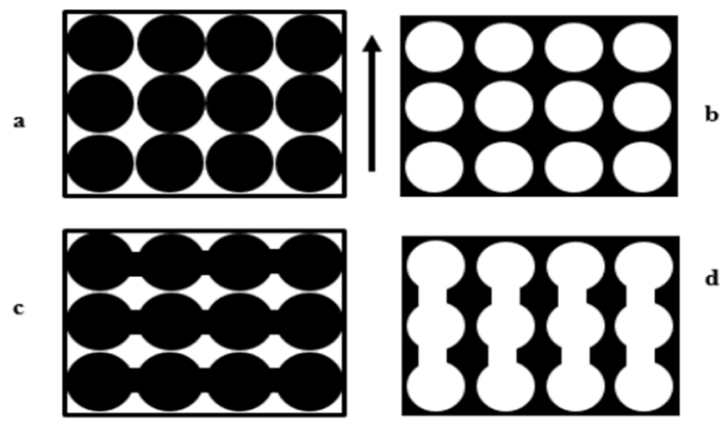

FIGURE 1. Schemes of the considered systems; a, b - initial structures, c, d - result of electric current action, black color - a weakly conductive phase, arrow - general direction of the current.

Apply to two opposite cell boundaries a dimensionless difference of electric potentials $\varphi$, equal to 0 at $y=0$ and at $1 \mathrm{y}=1$. The dimensionless conductivity of the matrix is assumed to be $\sigma \mathrm{m}=1$. The conductivities of the inclusions are $\sigma \mathrm{i}$. The surfaces $\mathrm{x}=0$ and $\mathrm{x}=1$ are assumed to be adiabatic.

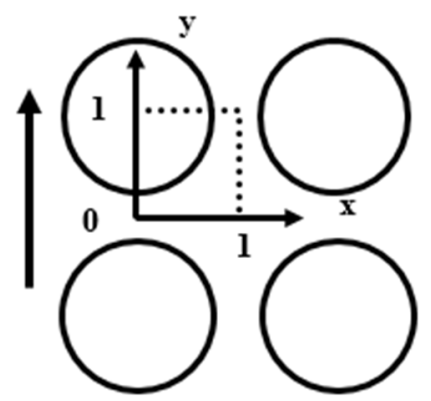

FIGURE 2. Unit cell diagram.

We divide the computational domain with round simplex elements [6]. The grid of partitions adapts to the boundary of inclusions [4]. We calculate the spatial distribution of the potential $\varphi(\mathrm{x}, \mathrm{y})$. For this purpose, we use the variation formulation of the transport equations, [6]. At the same time, we take into account the condition of externality of the functional:

$$
\chi=\int_{S_{c}} \sigma(\operatorname{grad} \varphi)^{2} d S
$$

Where Sc is the unit cell area, $\sigma$ is the local conductivity, $\mathrm{dS}$ is the area element of the computational domain.

The conditions of the minimum of the functional $\chi$ allow us to establish the nodal values of temperature at all points of the unit cell and, accordingly, the magnitudes of the gradients of the potential grad $(\varphi)$ and the Joule heat release density $\mathrm{q}=\sigma(\operatorname{grad}(\varphi))^{2}$.

To solve such a problem, a computer program in the FORTRAN language was created in the present work and a series of computational experiments were carried out, similar to the one described earlier [3-5].

\section{RESULTS OF COMPUTATIONAL EXPERIMENT}

In the process of calculations, the pictures of the spatial distributions $\mathrm{q}(\mathrm{x}, \mathrm{y})$ and the values of the effective conductivity $\sigma e f$ were analyzed for varying values of conductivity $\sigma \mathrm{i}$ and concentration of Ci inclusions. By us in a computational experiment, a triangulation of the calculated area with a frequency of $100 \times 100$ elements was used.

In Figure 3 the characteristic dependences of the relative density of heat release q / $\mathrm{q} 0$ are presented, where - $\mathrm{q}$ is local, and q0 is the average Joule heat density over the sample. The curves "a" and "d" in Fig. 3 are set with the ratio 
of the conductivities of the matrix and the inclusion $\sigma \mathrm{m} / \sigma \mathrm{i}=10: 1$ and 1:10, respectively; volume concentration of inclusions $\mathrm{Ci}=0.7$.

The systematization of the obtained data also allows us to conclude that the effects of concentration increase with an increase in the conductivity contrast $\sigma \mathrm{m} / \sigma \mathrm{i}$ and when Ci reaches critical values at which the particles touch.

It can be assumed that local overheating of the sintered material can lead to the appearance of "bridges" (connecting inclusions). In this case, two situations are realized, the schemes of which are shown in figures. 1c, d. If the compact phase has conductivity higher than the matrix, then conductive fibers are formed, oriented in the direction of the general flow of electric current. The effective conductivity of the material obtained acquires anisotropy of properties, which leads to increased electrical conductivity in the direction of current flow.

If the inclusions are weakly conductive in comparison with the matrix, then layers of sediment particles appear, perpendicular to the current axis. Anisotropy is characterized by a decrease in the effective conductivity in the direction of the heating current flow.

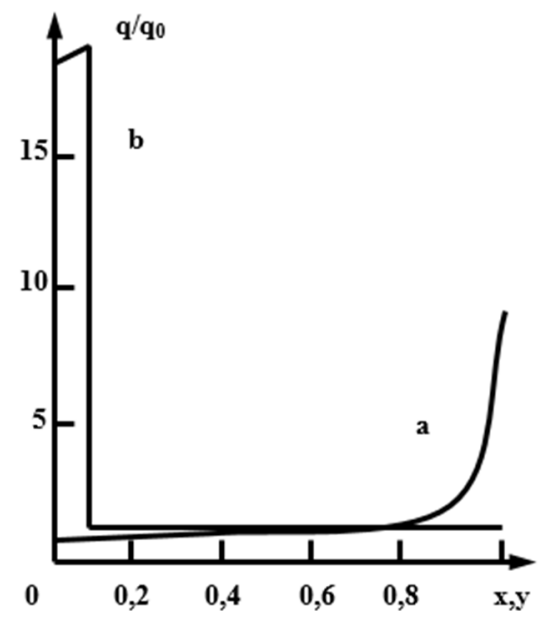

FIGURE 3. Dependences of the relative density of the Joule heat release, fixed along the $x$ y axes for structures a b, respectively.

In our opinion, the discussed features of the formation of anisotropy of material properties must be considered when using the method of synthesis using a flowing current.

It can also be suggested that the effects considered can also be used to create gradient materials [7 - 12].

\section{CONCLUSIONS}

1. When developing technologies for sintering powder materials by passing an electric current, it is necessary to take into account the substantial non-uniformity of Joule heat release.

2. The discussed effect of the concentration of heat release in the processes of the synthesis of powder materials by the method of passing an electric current can have a technological application for creating anisotropy of properties such as "selected axis" or "selected plane".

3. There is a fundamental possibility of creating gradient properties of materials by transmitting electric currents, in particular, when using microwave processing of multiphase materials.

\section{REFERENCES}

1. A.I. Raychenko, Basics of sintering powders by passing an electric current (1987) $128 \mathrm{p}$.

2. V.I. Ikryannikov, "The emergence of thermal structure in a non-uniform metallic conductor during the flow of high-density electric current", Journal of Engineering Physics and Thermophysics, Vol. 62, No. 4, pp. 617-623 (1992).

3. I.N. Sachkov, A.G. Hoffman, P.V.Geld, "Finite element method: conductivity and Joule heat generation in regular structures", News of Universities. Physics, 5, pp. 17-23 (1996). 
4. I.N. Sachkov. "The influence of the shape of inclusions on the conductivity of two-dimensional regular matrix systems", Technical Physics Journal, Vol. 66, №12. pp. 48-58. (1996).

5. V.T. Kuanishev, I.N. Sachkov, I.G. Sorogin and T.I. Sorogina, "The concentration parameter thermal microstresses as the thermophysical characteristics of two-phase materials", Journal of Physics: Conf. Series 891, 012354 (2017).

6. $\quad$ L. Segerlind, Applied Finite Element Analysis, 2nd edition (Wiley, New York, 1984), 427 p.

7. M.M. Gasik, Fundamentals of technology of anisotropic systems and functional gradient materials obtained by powder metallurgy methods, Dissert. Doctor of Technical Sciences, Helsinki, 234 p., 1999.

8. Sheppard L.M., "Innovative process for advanced ceramics", Amer. Ceram. Soc. Bull. 72, Vol 4, pp. 48-58, (1993).

9. M.M. Gasik, "Principles of functional gradient materials and their processing by powder metallurgy", Acta Polytechnica Scand, Vol. 226, 73 p. (1995).

10. Igor N. Sachkov, Viktoria F. Turygina, Maxim A. Medvedev, Aleksey N. Dolganov, and Kamen B. Spasov, "Development of new FEM programs for synergistic evaporation processes from porous systems", AIP Conference Proceedings 2040, 050017 (2018); https://doi.org/10.1063/1.5079115.

11. I.N. Sachkov, V.F.Turygina and A.N. Dolganov, "Kinetic synergistic transitions in the Ostwald ripening processes", IOP Conf. Series: Journal of Physics: Conf. Series 944, 012101 (2018); doi: 10.1088/17426596/944/1/012101.

12. Igor N. Sachkov, Dmitry B. Berg, Aleksey G. Gorman, Victoria F. Turygina and Oleg E. Khorev, "Optimization of the characteristics of three-phase resistive materials using finite element method", AIP Conference Proceedings 1863, 050012 (2017);https://doi.org/10.1063/1.4992209. 